\title{
A estratégia LET US Play aumenta a atividade física de crianças nas aulas de educação física escolar
}

\author{
The LET US Play strategy increases children`s physical activity in physical \\ education classes
}

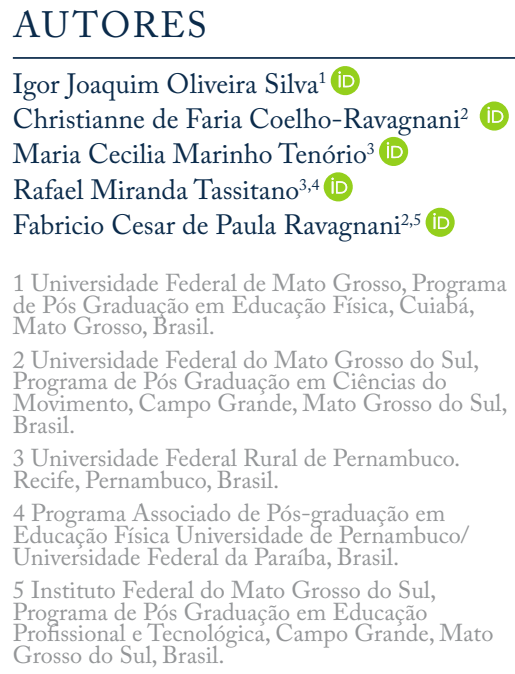

CONTATO

Igor Joaquim Oliveira Silva igorjoaquimassessoria@gmail.com

Faculdade de Educação Física, Universidade Federal de Mato Grosso.

Av. Fernando Corrêa da Costa, nº 2367, Bairro Boa Esperança. Cuiabá, Mato Grosso, Brasil.

CEP: 78060-900.

DOI

$10.12820 /$ rbafs. $26 \mathrm{e} 0238$

\section{(cc) BY}

Este trabalho está licenciado com uma Licença Creative Commons - Atribuiç̃o 4.0 Internacional.

\begin{abstract}
RESUMO
O estudo objetivou comparar duas estratégias (Tradicional x LET US Play) com o intuito de aumentar o número de passos (NP) durante as aulas de educação física escolar de crianças. Trata-se de um ensaio clínico randomizado cruzado (cross over), realizado em uma escola municipal de Cuiabá, Mato Grosso. Foram aplicadas 10 aulas à 25 crianças (11 meninos e 14 meninas) do $4^{\circ}$ ano do Ensino Fundamental. Dividida em dois momentos de 15 minutos (monitorados), com um espaço de 10 minutos entre eles para hidratação (não monitorados), a aula consistia na aplicação das atividades (estafeta, base quatro, futsal, queimada e empresta bandeira) usando a estratégia Tradicional ou LET US Play, de forma alternada e aleatória. Ao final de cada atividade, o NP foi medido por meio da pulseira inteligente Xiaomi Mi band 2. Utilizou ANOVA One Way para analisar o NP entre as estratégias, estratificada por sexo, com grau de significância de 5\%. Observou-se que, o NP dados pelas crianças na intervenção LET US Play foi superior ao Tradicional em todas as atividades (estafeta, base quatro, futsal, queimada, empresta bandeira), tanto em meninos quanto em meninas, independentemente da ordem de aplicação $(\mathrm{p}<0,01)$. Conclui-se que o LET US Play aumentou o NP das crianças durante as aulas de educação física e pode ser uma estratégia interessante para maximizar a atividade física nesse público.
\end{abstract}

Palavras-chave: Educação física; Atividade física; Jogos e brincadeiras; Pedometria; Dispositivo móvel.

\section{ABSTRACT}

This study aimed to compare two strategies (Traditional x LET US Play) focused on increasing the number of steps (NS) during physical education classes for children. This is a randomized crossover clinical trial, carried out in a municipal elementary school from Cuiabá-MT. Ten classes were given to 25 children (11 boys and 14 girls) from the 4 th grade. Divided into two moments of 15 minutes each (monitored), with a 10 minutes break between for hydration (not monitored). The class sessions consisted of activities (relay races, base four, futsal, dodgeball and borrow flag) using Traditional or LET US Play strategies, selected randomly and alternately. At the end of each activity the NS was measured through the Xiaomi Mi band 2 smart bracelet. The NP between the strategies and interactions with sex were assessed using one-way analysis of variance (ANOVA) with a statistical significance set at $p<0.05$. It was observed that the NS given by the children in the LET US Play intervention was higher than the Traditional in all activities (relay races, kickball, indoor soccer, dodgeball, lend flag) in boys and girls regardless of the delivered order $(p<0,01)$. It was concluded that LET US Play increased children's NS during physical education classes and can be an interesting strategy to maximize physical activity among this population.

Keywords: Physical activity; Games and play; Pedometry; Mobile device.

\section{Introdução}

A prática regular de atividade física (AF) traz benefícios fisiológicos, psicossociais e comportamentais (ex. melhora a saúde óssea, do condicionamento físico, reduz a ansiedade, controla o stress, melhora a autoestima, amplia os aspectos motores e cognitivos, etc), a curto ou a longo prazo $^{1-3}$. Contudo, apesar dos reconhecidos be- nefícios da $\mathrm{AF}$, dados de prevalência mundial reunindo 20 estudos em 10 países com mais de 27 mil crianças e adolescentes na faixa etária entre os 2,8 e 18,4 anos, sugerem que poucos jovens atingem as recomendações de atividade física para obter benefícios para a saúde ${ }^{3,4}$.

De acordo com Cooper e colaboradores apenas 9\% dos meninos e $1,9 \%$ das meninas, entre 5 a 17 anos, al- 
cançaram as recomendações de $\mathrm{AF}^{4}$. No Brasil, ainda há escassez de dados populacionais utilizando medidas objetivas, devido a limitações financeiras e logísticas ${ }^{5}$. Contudo, uma revisão sistemática apresentou, uma ampla variabilidade na prevalência de AF (6,5\% a 92,3\%) em crianças e adolescentes independentemente do instrumento utilizado (acelerometria, pedometria ou questionários), com altos índices de inatividade nas meninas ${ }^{5}$.

No intuito de aumentar a AF é importante que intervenções apropriadas à faixa etária sejam estabelecidas, especialmente aquelas voltadas aos grupos que apresentam menores níveis de atividade física (ex. meninas) $)^{6}$. A teoria das oportunidades expandidas (TEO) estabelece três mecanismos primários para a prática de AF, a saber: (a) Expansão, que se relaciona à inclusão de novas oportunidades de AF onde não existia; (b) Extensão, relacionada ao aumento da quantidade de tempo de uma oportunidade de AF já existente (c) Aprimoramento, ou seja, a melhoria da qualidade das oportunidades já existentes, por meio de estratégias que aumentem a AF acima da habitualmente praticada ${ }^{7}$.

As aulas de educação física (EF), componente curricular obrigatório na educação básica, são excelentes oportunidades para os jovens praticarem AF, sendo a participação nessas aulas uma grande contribuição nos níveis de AF diária dos escolares ${ }^{8,9}$. Entretanto, o tempo de aula não necessariamente se traduz em tempo de prática de $\mathrm{AF}$, especialmente de intensidade moderada a vigorosa ${ }^{10,11}$, o que compromete as aulas que contribuam para os objetivos de saúde ${ }^{12}$. A oferta de apenas uma a duas aulas de EF por semana, como ocorre na maioria das escolas brasileiras, constitui-se mais um limitador para a promoção da prática de $\mathrm{AF}$ dos estudantes ${ }^{13}$. Considerando a dificuldade para aumentar a frequência semanal de aulas de EF entende-se ser necessário aprimorar as aulas existentes, incrementando o envolvimento dos jovens na $\mathrm{AF}$ e, consequentemente, o tempo que os mesmos dispendem em atividade física moderada a vigorosa (AFMV) ${ }^{14}$.

O LET US Play, com tradução livre "Vamos Jogar", é uma estratégia desenvolvida por Weaver e colaboradores em $2013^{15}$, que visa maximizar a AF, especialmente de intensidade moderada a vigorosa, de crianças e adolescentes, propondo a adaptação de esportes, brincadeiras e jogos tradicionais, podendo ser usada em diversos contextos como escolinhas de esporte e na EF escolar $^{16}$. Sua sigla em inglês corresponde às principais barreiras à $\mathrm{AF}$ em crianças e adolescentes durante as atividades ou jogos: Lines - filas; Elimination - eli- minação; Team size - tamanho da equipe; Univolved staff/kids - professores e alunos não envolvidos; $\underline{\text { Space, }}$ equipment and rules - espaço, equipamentos e regras ${ }^{1}$. Essas barreiras, quando identificadas, são modificadas. Assim, o LET US Play guarda relação com a TEO, embasando-se na participação ativa dos professores, no uso de materiais diversos e na diversidade de jogos ${ }^{15-18}$.

No entanto, apesar das bases teóricas que cercam essa proposta, o método nunca foi testado durante as aulas de EF escolar no Brasil e em nenhum outro país ${ }^{15-18}$. Além disso, são poucos os estudos sobre programas de intervenção em promoção da AF e saúde entre os escolares brasileiros ${ }^{19}$. Nesse sentido, mediante a relevância do contexto (aulas de EF) e da estratégia de intervenção (LET US Play), este trabalho é o primeiro a utilizar o LET US Play dentro do ambiente escolar. Sendo assim, este estudo objetiva comparar duas estratégias de ensino (tradicional x LET US Play) para aumentar o número de passos dos escolares durante as aulas de Educação Física, considerando as diferenças entre meninos e meninas.

\section{Métodos}

O estudo foi aprovado pelo Comitê de Ética em Pesquisa com Seres Humanos da Universidade Federal de Mato Grosso (n.10037219.3.0000.8124). Todos os participantes retornaram com o termo consentimento livre e esclarecido assinado pelos responsáveis, leram e assinaram o termo de assentimento para crianças.

Foi realizado um estudo de intervenção, tipo ensaio clínico randomizado, cruzado (cross-over), onde os participantes foram submetidos a duas condições de intervenções: atividades com regras originais (tradicional) e atividades com as regras modificadas (LET US Play).

A população foi constituída por escolares com idade entre 9 e 12 anos, matriculados no Ensino Fundamental de uma escola municipal da zona urbana de Cuiabá, Mato Grosso, seguindo uma amostragem por conveniência. A instituição possuía, no período da pesquisa, 100 alunos matriculados nos anos iniciais, sendo 4 turmas no ensino fundamental ( $1^{\circ}$ ao $4^{\circ}$ ano).

Após contato e permissão da direção e professores da escola, das 04 turmas existentes (total de 100 alunos matriculados), apenas o professor responsável da turma do $4^{\circ}$ ano do ensino fundamental demonstrou interesse em participar da pesquisa. A classe, com seus 25 escolares (11 meninos e 14 meninas) com idade média de 9 anos, foram considerados elegíveis para participarem do estudo.

Para participar, adotaram-se os seguintes critérios 
de inclusão: interesse e disponibilidade em frequentar regularmente as atividades de intervenção, duas vezes por semana durante quatro meses. Foram excluídos da análise os dados coletados de um aluno PCD (Pessoa com Deficiência) que fazia uso de cadeira de rodas. Vale frisar que, compreendendo o processo de inclusão no sistema de ensino o aluno participou das atividades normalmente, contudo para efeito de estudo seus dados não foram utilizados.

Informações como idade, sexo, local de residência, etnia, nível de escolaridade e renda, foram obtidas por meio de um questionário sóciodemográfico preenchido pelo responsável do participante.

A intervenção ocorreu no período de junho a setembro de 2019, na quadra poliesportiva da instituição, durante 16 semanas, (terças e quintas) das 8 às $9 \mathrm{~h}$ da manhã, nos dias que não havia aula de Educação Física para turma, respeitando o cronograma da escola.

As intervenções totalizaram 10 aulas, todas coordenadas pelo pesquisador (professor de educação física sem vínculo com a instituição), com suporte do professor de sala (pedagogo responsável pela turma). Cada aula teve duração de 50 minutos e foram estruturadas conforme Quadro 1.

Foram utilizados jogos populares da cultura brasileira, sendo três selecionados de forma aleatória pelo pesquisador: base 04, estafeta, empresta bandeira e dois indicados pelos escolares: queimada e futsal. Para definir as estratégias de modificação das regras dos jogos levou-se em consideração o espaço de aplicação, materiais disponíveis e número de participantes. As descrições dos jogos, estratégias de implementação e regras originais (tradicional) e modificadas (LET US Play), são apresentados no Quadro 2.

A aula foi dividida em dois momentos de $15 \mathrm{mi}^{-}$ nutos, sendo que os jogos foram aplicados por igual número de vezes durante o primeiro ou segundo momento. Durante as aulas, os participantes não eram informados sobre a estratégia que estava sendo utilizada (tradicional ou LET US Play), havia apenas uma explicação sobre as regras do jogo que seria aplicada naquele momento (primeiro ou segundo).

A coleta iniciava-se em sala de aula, onde os alunos recebiam orientações sobre as atividades programadas e formas de uso das pulseiras. $\mathrm{Na}$ quadra as crianças recebiam mais informações, eram divididas em equipes (aleatório) e tinham suas pulseiras zeradas, ocorrendo o mesmo procedimento no início de cada momento. Ao término de cada momento o número de passos era anotado. Antes de retornar para a sala de aula, as pulseiras eram recolhidas pelo pesquisador.

Antes do período de intervenção, foram realizadas medidas antropométricas de peso e estatura. As mesmas ocorreram dentro da sala de aula com os escolares vestidos com o uniforme do colégio. $O$ peso foi mensurado por meio de uma balança de vidro $\left(\right.$ Techline $\left.^{\circledR}\right)$ com capacidade de até $150 \mathrm{~kg}$. A estatura foi medida por uma fita métrica inelástica, com $1,50 \mathrm{~m}$ de comprimento.

Para a pesagem os alunos foram orientados a ficarem descalços na posição ortostática (em pé e corpo ereto) braços soltos lateralmente ao corpo, ombros descontraídos e mantendo a cabeça erguida e olhando para frente. Para a estatura utilizou-se uma fita métrica fixada na parede da sala de aula, com campo de medição de $200 \mathrm{~cm}$. O participante foi posicionado com os braços ao longo do corpo, pés unidos e cabeça, nádegas e calcanhares encostados na parede, descalços e com a cabeça posicionada no plano de Frankfurt. A leitura foi realizada em apnéia após uma expiração normal. $\mathrm{O}$ Índice de Massa Corporal foi calculado pela equação

Quadro 1 - Apresentação dos jogos/brincadeiras, duração e a ordem das intervenções.

\begin{tabular}{|c|c|c|c|c|c|c|}
\hline Aula & Jogos/brincadeiras & $\begin{array}{l}\text { Início } \\
5 \text { min }\end{array}$ & $1^{\circ}$ momento $15 \mathrm{~min}$ & $\begin{array}{c}\text { Intervalo } \\
10 \mathrm{~min}\end{array}$ & $\begin{array}{l}2^{\circ} \text { momento } \\
15 \text { min }\end{array}$ & $\begin{array}{c}\text { Fim } \\
5 \mathrm{~min}\end{array}$ \\
\hline 01 & Estafeta & \multirow{10}{*}{$\begin{array}{c}\text { Colocação da } \\
\text { pulseira. }\end{array}$} & Tradicional & \multirow{10}{*}{$\begin{array}{l}\text { Coleta de dados, } \\
\text { pausa para água, } \\
\text { reinicialização da } \\
\text { pulseira }\end{array}$} & LET US Play & \multirow{10}{*}{$\begin{array}{c}\text { Remoção da } \\
\text { pulseira }\end{array}$} \\
\hline 02 & Base 04 & & LET US Play & & Tradicional & \\
\hline 03 & Queimada & & Tradicional & & LET US Play & \\
\hline 04 & Futsal & & LET US Play & & Tradicional & \\
\hline 05 & Empresta bandeira & & Tradicional & & LET US Play & \\
\hline 06 & Estafeta & & LET US Play & & Tradicional & \\
\hline 07 & Base 04 & & Tradicional & & LET US Play & \\
\hline 08 & Queimada & & LET US Play & & Tradicional & \\
\hline 09 & Futsal & & Tradicional & & LET US Play & \\
\hline 10 & Empresta bandeira & & LET US Play & & Tradicional & \\
\hline
\end{tabular}

Min: minutos. 
Quadro 2 - Descrições dos jogos, estratégias de implementação e regras originais (Tradicional) e modificadas (LET US Play)

\begin{tabular}{|c|c|c|c|}
\hline Jogo & Descrição & Princípios Usados & Modificações \\
\hline Base 04 & $\begin{array}{l}\text { - Duas equipes (Rebatedores e Receptores); } \\
\text { - Rebatedores posicionam em fila; } \\
\text { - Realiza a corrida entre as bases } 1 \text { rebatedor por } \\
\text { vez; } \\
\text { - Receptores disperso na quadra com um } \\
\text { integrante dentro do círculo central (esse não pode } \\
\text { sair do centro); } \\
\text { - Receptores devem pegar a bola rebatida e levar } \\
\text { ao aluno no círculo central; } \\
\text { - O rebatedor é eliminado quando, fora da base, a } \\
\text { bola chega até o receptor central; } \\
\text { - Após todos rebatedores terem rebatidos as } \\
\text { equipes mudam de função; } \\
\text { - Vence a equipe que acumular mais pontos. }\end{array}$ & $L-E-U-S$ & $\begin{array}{l}\text { - Duas equipes (Rebatedores e Receptores); } \\
\text { - Rebatedores disperso na linha de fundo; } \\
\text { - Realiza a corrida entre as bases todos os rebatedores; } \\
\text { - Receptores disperso na quadra; } \\
\text { - Receptores devem pegar a bola e todos se reunirem no } \\
\text { círculo central; } \\
\text { - Os rebatedores devem retornar a linha de fundo } \\
\text { quando os receptores chegarem ao meio; } \\
\text { - Após todos rebatedores terem rebatidos, as equipes } \\
\text { mudam de função; } \\
\text { - Vence a equipe que acumular mais pontos. }\end{array}$ \\
\hline $\begin{array}{l}\text { Empresta } \\
\text { Bandeira }\end{array}$ & $\begin{array}{l}\text { - Uma quadra } \\
\text { - Duas grandes equipes; } \\
\text { - Uma bandeira por equipe (na linha de fundo); } \\
\text { - Jogador colado no campo de ataque deve ficar } \\
\text { parado no lugar até que alguém (da sua equipe) o } \\
\text { descole; } \\
\text { - Vence a equipe que trazer a bandeira para o seu } \\
\text { campo. }\end{array}$ & $E-T-U-S$ & $\begin{array}{l}\text { - Várias mini quadra; } \\
\text { - Duas equipes (reduzida) em cada mini jogo; } \\
\text { - Três bandeiras por equipe em cada mini jogo; } \\
\text { - Jogador pego no campo de ataque, para voltar a atacar, } \\
\text { deve retornar para o campo de defesa. } \\
\text { - Vence a equipe que tiver o maior número de } \\
\text { bandeiras. }\end{array}$ \\
\hline Queimada & $\begin{array}{l}\text { - Uma quadra; } \\
\text { - Duas grandes equipes; } \\
\text { - Uma bola; } \\
\text { - Jogador queimado sai do jogo (deve esperar o } \\
\text { jogo terminar para voltar); } \\
\text { - Vence a equipe que eliminar todos os integrantes } \\
\text { da outra equipe. }\end{array}$ & $E-T-U-S$ & $\begin{array}{l}\text { - Várias mini quadra; } \\
\text { - Duas equipes (reduzida) em cada mini jogo; } \\
\text { - Duas bolas por mini jogo; } \\
\text { - Jogador queimado troca de equipe; } \\
\text { - Vence a equipe que tiver o maior número de } \\
\text { integrantes. }\end{array}$ \\
\hline Estafeta & $\begin{array}{l}\text { - Duas filas; } \\
\text { - Grandes equipes; } \\
\text { - Um aluno por vez executa a corrida; } \\
\text { - Vence a equipe que completar os jogos de } \\
\text { velocidade primeiro. }\end{array}$ & $\mathrm{L}-\mathrm{T}-\mathrm{U}-\mathrm{S}$ & $\begin{array}{l}\text { - Seis filas; } \\
\text { - Equipes menores; } \\
\text { - Todos correm simultaneamente; } \\
\text { - Vence a equipe que completar os jogos de velocidade } \\
\text { primeiro. }\end{array}$ \\
\hline Futsal & $\begin{array}{l}\text { - Uma quadra; } \\
\text { - Dois times; } \\
\text { - Duas traves (tamanho oficial) } \\
\text { - Cinco jogadores por equipe; } \\
\text { - Um goleiro por equipe; } \\
\text { - A equipe vencedora permanece na quadra; } \\
\text { - A equipe derrotada aguarda sentado até o } \\
\text { término da partida. }\end{array}$ & $E-T-U-S$ & $\begin{array}{l}\text { - Várias mini quadra; } \\
\text { - Duas equipes por mini quadra; } \\
\text { - Duas traves por mini jogo (tamanho reduzido); } \\
\text { - Três jogadores por equipe; } \\
\text { - Não há goleiro; } \\
\text { - Todos jogam ao mesmo tempo. }\end{array}$ \\
\hline
\end{tabular}

Fonte: Adaptado do estudo de Weaver; Webster; Beets ${ }^{15} . \mathrm{L}=$ line (remoção ou redução das filas durante os jogos/brincadeiras); $\mathrm{E}=$ elimination (retirar da regra do jogo a eliminação de um participante); $\mathrm{T}=$ teamsize (tamanho da equipe, reduzir os tamanhos das equipes formando vários mini-jogos); $\mathrm{U}=$ uninvolved staff (maior envolvimento dos professores com os alunos durante as atividades); $\mathrm{S}=$ space, equipment, rules (utilizar maior espaço, mais equipamentos e modificar as regras do jogo).

peso/estatura ${ }^{2}$ utilizando os critérios da Organização Mundial da Saúde para classificá-los em desnutrição, eutrófico, sobrepeso e obeso ${ }^{20}$.

A contagem de passos foi feita com auxílio de dispositivos vestíveis, as pulseiras inteligente da marca Xiaomi Mi Band 2, com tela OLED, retroiluminação, sensíveis ao toque, bluetooth, sensores (acelerômetros e medidor de batimentos cardíacos), feitas de borracha e resistentes a água. $\mathrm{O}$ dispositivo, em termos de precisão e estabilidade da medição do número de passos, apresenta um desempenho satisfatórios com média absoluta de erro percentual de 0,06 (Dp =0,17), mostrando ser capaz de medir com segurança o número de passos, podendo ser usada como indicador efetivo de avaliação da saúde ${ }^{21}$.

O aparelho era anexado no pulso esquerdo da criança e zerada antes do início de cada momento de atividade. Ao término de cada momento anotava-se o número de passos despendido na atividade. Para verificar o percentual de tempo em AFMV, adotou-se 60,6 e 82,2 passos/minuto como pontos de corte, visto que esses valores estão estabelecidos na literatura e correspondem a executar $33 \%$ e $50 \%$ do tempo da aula em AFMV respectivamente ${ }^{22}$.

Para a verificação da normalidade dos dados foi uti- 
lizado o teste de Shapiro-Wilk. Os dados apresentaram distribuição normal, assim os números de passos foram expressos em média $(\bar{x})$ e desvio padrão $(\mathrm{Dp})$, divididos por estratégia de aulas (tradicional e LET US Play), momento da atividade (primeiro ou segundo), gênero (masculino e feminino) e atividades (estafeta, base 04, queimada, futsal, empresta bandeira).

Para analisar a influência do momento da atividade no número de passos em cada estratégia e o número de passos entre as estratégias, utilizou a análise de variância de medidas repetidas (ANOVA) com post hoc de Tukey. $O$ nível de significância aceito foi de $p \leq 0,05$. $O$ tratamento estatístico foi realizado pelo software Instat 3.

\section{Resultados}

Participaram do presente estudo 25 estudantes (11 meninos e 14 meninas) de 9 e 10 anos de idade matriculados no $4^{\circ}$ ano do ensino fundamental de uma escola municipal da cidade de Cuiabá, Mato Grosso. Dos 25 participantes $12 \%$ estavam abaixo do peso, $64 \%$ eram eutróficos, $16 \%$ obesos e $8 \%$ apresentaram sobrepeso. Fatores como ausência, mal-estar e desinteresse, recusa de participação em um dos momentos e disponibilidade de aparelhos, influenciaram no número de participantes em cada aula, que variou entre 17 a 20 alunos.

A Figura 1 apresenta as médias do número de passos das crianças nos jogos praticados no formato tradicional e LET US Play, em diferentes momentos (primeiro ou segundo). Observa-se que o número de passos dados pelas crianças, ou seja, os passos acumulados, foram superiores quando os jogos são modificados com os princípios LET US Play.

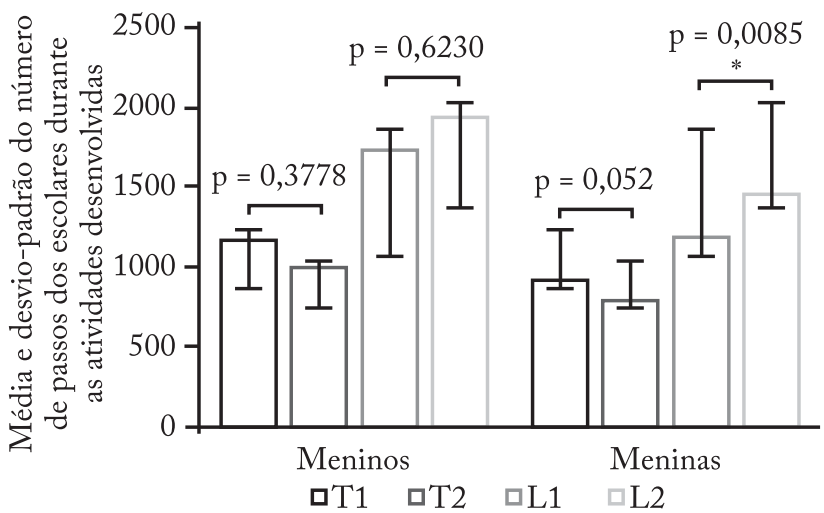

Figura 1 - Análise da média e desvio padrão dos passos acumulados pelos meninos e meninas nos jogos (base 04, estafeta, empresta bandeira, futsal, queimada) aplicados em dois momentos de 15 minutos cada, com estratégias diferentes: tradicional (T) ou LET US Play (L). $\mathrm{T} 1=$ tradicional $1^{\circ}$ momento; $\mathrm{T} 2=$ tradicional $2^{\circ}$ momento; L1= Let Us Play $1^{\circ}$ momento; L2= Let Us Play $2^{\circ}$ momento. *diferença significante entre T1 e T2 e L1 e L2.
A comparação das médias do número de passos dos meninos e das meninas de acordo com o jogo praticado e estratégia utilizada são apresentadas na Tabela 1. Foram observadas médias de passos superiores, tanto em meninos quanto em meninas, nos jogos modificados com a estratégia LET US Play $(\mathrm{p}<0,05)$.

Tabela 1 - Média e desvio padrão dos passos durante as atividades de 15 minutos separados por gênero (masculino e feminino) e estratégia (tradicional e LET US Play)

\begin{tabular}{|c|c|c|c|c|}
\hline \multirow{3}{*}{ Jogo } & \multicolumn{3}{|c|}{ Meninos } & \multirow{3}{*}{$\mathrm{p}$} \\
\hline & \multirow{2}{*}{$\mathrm{n}$} & Tradicional & LET Us Play & \\
\hline & & $\bar{x} \pm \mathrm{DP}$ & $\bar{x}_{ \pm \mathrm{DP}}$ & \\
\hline Estafeta & 17 & $818,35 \pm 435,69$ & $1442,29 \pm 759,23^{*}$ & $<0,01$ \\
\hline Base 04 & 15 & $903,13 \pm 348,67$ & $1670,80 \pm 765,43^{*}$ & $<0,01$ \\
\hline Queimada & 17 & $1184,88 \pm 857,72$ & $2136,12 \pm 1332,31^{*}$ & $<0,01$ \\
\hline Futsal & 13 & $1091,92 \pm 384,67$ & $2045,15 \pm 1208,34^{*}$ & $<0,01$ \\
\hline \multirow[t]{2}{*}{$\begin{array}{l}\text { Empresta } \\
\text { bandeira }\end{array}$} & 16 & $1417,81 \pm 589,80$ & $1892,25 \pm 783,17^{*}$ & $<0,01$ \\
\hline & \multicolumn{3}{|c|}{ Meninas } & \\
\hline \multirow[t]{2}{*}{ Jogo } & $\mathrm{n}$ & Tradicional & LET Us Play & $\mathrm{p}$ \\
\hline & & $\bar{x}_{ \pm \mathrm{DP}}$ & $\bar{x}_{ \pm \mathrm{DP}}$ & \\
\hline Estafeta & 23 & $670,74 \pm 324,63$ & $1076,04 \pm 385,50^{*}$ & $<0,01$ \\
\hline Base 04 & 22 & $793,68 \pm 325,33$ & $1178,27 \pm 502,15^{*}$ & $<0,01$ \\
\hline Queimada & 22 & $941,45 \pm 361,41$ & $1378,55 \pm 513,23^{*}$ & $<0,01$ \\
\hline Futsal & 23 & $828,04 \pm 457,82$ & $1395,57 \pm 639,25^{*}$ & $<0,01$ \\
\hline $\begin{array}{l}\text { Empresta } \\
\text { bandeira }\end{array}$ & 22 & $1031,95 \pm 455,88$ & $1569,45 \pm 723,46^{*}$ & $<0,01$ \\
\hline
\end{tabular}

*Diferença significante entre o método Tradicional e LET US Play em meninos e meninas $(\mathrm{p}<0,05)$. Teste ANOVA One Way. $\bar{x}=$ Média; DP = Desvio padrão; $\mathrm{n}=$ Número de participantes.

Quando analisado o número de passos por minuto durante as atividades (tradicional vs LET US Play) (Figura 2), seguindo as recomendações de 82,20 passos/minutos o que caracteriza $50 \%$ do tempo da aula em AF moderada a vigorosa, se evidenciou que o $L E T$ US Play, em comparação ao tradicional, foi capaz de aumentar as médias dos passos por minutos tanto em meninos $(121,50 \pm 18,02)$ quanto em meninas $(87,90$ $\pm 12,91)$.

\section{Discussão}

Este estudo é o primeiro a avaliar a efetividade da estratégia LET US Play no aumento da AF das crianças brasileiras durante as aulas de EF escolar. Os resultados indicam que todos os jogos/brincadeiras modificados, segundo os princípios LET US Play, proporcionaram aumento no número de passos, tanto em meninos quanto em meninas, independente da ordem em 


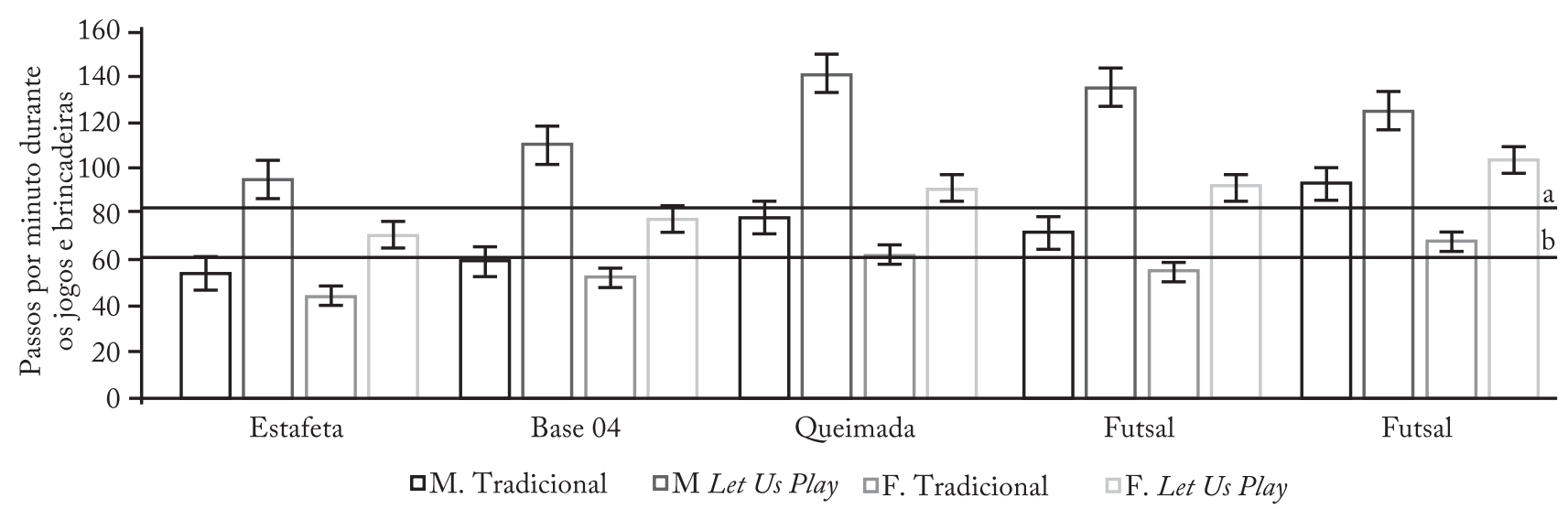

Figura 2 - Média e desvio padrão dos passos/minuto atingidos pelos meninos (M) e meninas (F), durante os 15 minutos de jogos/brincadeiras aplicados seguindo a estratégia tradicional e a LET US Play. A = recomendação de passos/minuto $(82,20)$ que representa $50 \%$ do tempo da aula em AFMV; b = recomendação de passos/minuto $(60,60)$ que representa 33\% do tempo da aula em AFMV.

que a intervenção foi aplicada (primeiro ou segundo momento da aula). Ademais, a estratégia aumentou o número de passos por minuto de 68 a 54\%, em ambos os sexos, tornando possível atingir, superar ou aproximar-se das recomendações dos 82,2 passos $/ \mathrm{min}^{22}$.

Maiores quantidades de AFMV na juventude geram maior benefício físico, emocional, individual, social, intelectual e financeiro ${ }^{23}$ a curto e a longo prazo $^{22}$. Em contrapartida, atividades leves ainda não têm estabelecido o efeito dose-resposta para muitas condições clínicas $^{24}$ e está relacionada com o aumento progressivo do tempo sedentário com o passar da idade ${ }^{4}$. Nesse sentido, a utilização de estratégias que possibilitam o aumento do tempo em AFMV se tornam indispensáveis na promoção da saúde, ratificando a relevância dos achados dessa pesquisa.

Nesse contexto, analisando o tempo de permanência em AFMV durante as atividades, os resultados do presente estudo se alinham com os do estudo de Brazendale $^{18}$, que aplicou a estratégia LET US Play com acelerômetros, em um grupo de crianças, durante um acampamento de verão, fora do ambiente escolar e, evidenciou um aumento de $25 \%$ do tempo em AF moderada a vigorosa tanto para meninos quanto para meninas ${ }^{18}$. Ao aplicar o LET US Play com pedômetros, no contexto brasileiro, dentro do ambiente escolar, este estudo observou um aumento similar para ambos os sexos, adicionando novas informações sobre a estratégia. Além disso, a pesquisa apresentou contadores de passos como instrumento promissor para otimização da AF nas aulas de EF, sendo bons estimuladores para a prática de AF dos alunos dentro e fora da escola ${ }^{22}$. Vale frisar que, neste estudo, no intuito de minimizar a interferência da nova ferramenta no número de passos, foi realizado um procedimento de familiarização dos alunos com os pedômetros (apresentação em sala e atividades práticas), iniciando as atividades programadas após a turma estar ambientada com o dispositivo, minimizando dessa forma a reatividade ao aparelho.

É possível observar também, o aumento do número de passos dos alunos em todas as atividades aplicadas com a estratégia LETUS Play, com médias maiores nos meninos do que nas meninas. Fatores como confiança nas próprias habilidades, capacidade de arriscar-se ${ }^{25}$ dependência tradicional do jogo, de forma organizada e competitiva contribuem para baixa efetividade das meninas, quando comparado aos meninos, durante as aulas de EF escolar ${ }^{26}$. Ciente da preferência de alguns escolares pela $\mathrm{AF}$ não competitiva ${ }^{26,27}$ e da influência do comportamento habitual durante a infância na vida adulta $^{6}$ entende-se como imprescindível a busca por novas estratégias para otimização das aulas de EF escolar, possibilitando assim uma aula que inclua o maior número de participantes.

No que tange aos princípios utilizados, os jogos que tiveram em comum a retirada da eliminação do participante apresentaram médias de passos superiores em relação às demais atividades. De fato, a eliminação impossibilita que o tempo de aula se traduza em tempo em $\mathrm{AF}^{12}$, fazendo com que o aluno fique inativo parte da aula para fazer cumprir as determinações impostas pelo jogo ${ }^{15,16}$. Assim, os escolares nesses momentos são induzidos a buscar atividades sedentárias, diminuindo o tempo de prática gerando insatisfação, perda de vontade e busca por outras atividades.

Por fim, ao implementar os jogos e brincadeiras tradicionais utilizando a estratégia LET US Play o profissional da EF desenvolve, a partir de um jogo, uma 
infinidade de brincadeiras, possibilitando o aumento das oportunidades de prática e, consequentemente, o aumento do nível de AF. Isso economiza tempo, por exemplo, aprendendo um novo jogo e diminui a interrupção do funcionamento rotineiro das aulas de $\mathrm{EF}^{18}$. Todavia, cabe ao profissional fazer uso dos princípios, levando em consideração a turma, o ambiente e os materiais. Dessa forma, durante a elaboração das atividades, o professor deve, a partir da regra original da brincadeira que será proposta, identificar as barreiras que impeçam a participação ativa das crianças, considerar o ambiente/material disponível para prática, modificando, se necessário, as regras segundo os princípios da estratégia.

Dentre as limitações deste estudo destacam-se: amostragem por conveniência, qualidade psicométricas do instrumento utilizado e a não análise de outros desfechos (participação, motivação, etc). $\mathrm{O}$ cálculo da amostra não foi realizado por uma questão operacional, pois havia uma amostra disponível. Os avaliados representavam uma turma de uma escola, e portanto, a generalização desses achados pode ser limitada a outras configurações (por exemplo, escolares de outras faixa etárias). Sobre o instrumento, apresenta boa validade em grupos etários maiores ${ }^{21}$, necessitando de mais estudos brasileiros. Em relação às aulas, percebe-se a necessidade de analisar outros desfechos relevantes em uma aula de EF (motivação, participação, aprendizado, etc). Independentemente disso, o protocolo aqui estabelecido fornece um modelo para ser replicado em amostras maiores e mais representativas.

Conclui-se que a implementação dos jogos tradicionais utilizando a estratégia LET US Play proporciona o aumento do número de passos, em meninos e meninas do $4^{\circ}$ ano do ensino fundamental de uma escola municipal, durante as aulas de EF escolar. Para tanto, este trabalho adicionou mais informações sobre a aplicabilidade da estratégia LET US Play, no contexto da EF escolar do Brasil.

Incentivam-se mais estudos sobre a estratégia nas aulas de EF em crianças e adolescentes nas redes de ensino público e privada, a fim de validar a eficiência da estratégia. São necessárias pesquisas que também explorem as atividades físicas em outros contextos. Recomenda-se também, estudos que utilizem a estratégia em uma amostra randomizada.

\section{Conflito de interesse}

Os autores declaram não haver conflito de interesse.

\section{Contribuição dos autores}

Silva IJO, participou de todas as etapas da pesquisa construção do projeto, coleta de dados, análise e escrita do artigo. Ravagnani FCP, participou de todas as etapas da pesquisa, orientação e escrita do artigo. Coelho-Ravagnani CF, contribuiu com o delineamento da pesquisa, redação do artigo e revisão crítica. Tenorio MCM e Tassitano RM, contribuiram com a redação do artigo, análise estatística e revisão crítica.

\section{Agradecimentos}

À Universidade Federal de Mato Grosso e Mato Grosso do Sul, aos grupos de pesquisa PENSARE e ESTIC, a EMEB Maria Lucila da Silva Barros Barros, ao professor PHD. R. Glenn Weaver e ao professor Luis Carlos de Oliveira.

\section{Referências}

1. Moreira RB. Níveis de atividade física nas aulas de educação física [tese de doutorado]. Porto Alegre: Universidade Federal do Rio Grande do Sul; 2014.

2. Hallal PC, Cesar GV, Mario RA, Jonathan CKW. Adolescent physical activity and health: a systematic review. Sports Med. 2006;36(12):1019-30.

3. Telama R. Tracking of physical activity from childhood to adulthood: a review. Obes Facts. 2009;3:187-95.

4. Cooper AR, Goodman A, Page AS, Sherar LB, Esliger DW, Sluijs EMF, et al. Objectively measured physical activity and sedentary time in youth: the international children's accelerometry database (ICAD). Int $\mathrm{J}$ BehavNutrPhy. 2015;12(113):1-10.

5. Filho VCB, Costa RM, Knebel MTG, Oliveira BN, Silva CBA, Silva KS. The prevalence of global physical activity among Young people: a systematic review for the report card Brazil 2018. Rev Bras Cineantropom Hum. 2018;20(4):367-87.

6. Kwon S, Kathleen FJ. Tracking of accelerometry-measured physical activity during childhood: ICAD pooled analysis. Int J Behav Nutr Phy. 2012;9(68):1-8.

7. Beets MW, Okely A, Weaver RG, Webster C, Lubans D, Brusseau T, et al. The theory of expanded, extended, and enhanced opportunities for youth physical activity promotion. Int J Behav Nutr Phy. 2016;13(120):1-15.

8. Turdor-Loke C, Lee SM, Morgan CF, Beighle A, Pangarazi RP. Morgan CF, Pangarazi RP, Beighle A. Children's pedometer-determined physical activity during the segmented school day. Med Sci Sports Exerc. 2006;38(10):1732-8.

9. Soares CAM, Hallal PC. Interdependência entre a participação em aulas de Educação Física e níveis de atividade física de jovens brasileiros: estudo ecológico. Rev Bras Ativ Fís Saúde. 2015;20(6):588-90.

10. Carlson JA, Sallis JF, Chriqui JF, Schneider L, Mcdermid LC, Agron P. State policies about physical activity minutes in physical education or during school. J Sch Health. 2013;83(3):150-6.

11. IBOPE. Relatório de pesquisa Educação Física nas escolas públicas brasileiras: 2012. São Paulo: IBOPE; 2012 [citado em 2020 jul 07]. Disponível em: http://cev.org.br/arquivo/ biblioteca/educacao-fisica-nas-escolas-publicas-brasileirasrelatorio-pesquisa.pdf. 
12. UNESCO.Diretrizes em educação física de qualidade (EFQ) para gestores de politica: 2015. Brasília: UNESCO. [citado em 2020 set 1]. Disponível em: http://unescoittralee.com/ wp-content/uploads/2017/11/QPE-for-policy-makersPortuguese.pdf.

13. IBGE. Pesquisa nacional de saúde escolar: 2015. Rio de Janeiro: IBGE; 2016. [citado em 2020 set 01]. Disponível em: https://biblioteca.ibge.gov.br/visualizacao/livros/ liv97870.pdf.

14. SBP. Promoção da atividade física na infância e adolescência: 2017. Rio de Janeiro: SBP; 2017. [citado em 2020 set 01]. Disponível em: https:/www.sbp.com.br/fileadmin/ user_upload/19890e-MO-Promo_AtivFisica_na_Inf_e_ Adoles-2.pdf.

15. Weaver RG, Webster C, Beets MW. Let us play: maximizing physical activity in physical education. Strategies. 2013;26(6):33-7.

16. Ravagnani FCP,Alexandre MG, Ravagnani CFC, Brazendale $\mathrm{K}$, Weaver R, Beets M. Usando os princípios let us play para maximizar a atividade física em aulas de educação física. Thema. 2018;15(4):1493-98.

17. Ickes MJ, Erwin H, Beighle A. Systematic review of recess interventions to increase physical activity. J Phys Act Health. 2013;10:910-26.

18. Brazendale K, Chandler JL, Beets MW, Weaver RG, Beighle A, Huberty JL, et al. Maximizing children's physical activity using the let us play principles. PM. 2015;76:14-9.

19. Souza EA, Barbosa Filho VC, Nogueira JAD, Azevedo MR. Atividade física e alimentação saudável em escolares brasileiros: revisão de programas de intervenção. Cad de Saúde Pública.2011;27(8):1459-71.
20. World Health Organization (WHO).Adolescent obesity and related behaviours : Observations from the Health Behaviour. World Health Organization Regional Office For Europe; 2017. [Citado em 2020 jul 07]. Disponível em: https:/www. euro.who.int/_data/assets/pdf_file/0019/339211/WHO_ ObesityReport_2017_v3.pdf?ua=1.

21. Xie J, Wen D, Liang L, Jia Y, Gao L, Lei J. Evaluating the validity of current mainstream wearable devices in fitness tracking under various physical activities: Comparative study. JMIR. 2018;6(4):1-21.

22. Scruggs PW. Quantifyng physical activity in physical education via pedometry: a further analysis of steps $/ \mathrm{min}$ guidelines. J Phys Act Health. 2013; 10; 734-41.

23. Lisa M, Gopu N, Howson N. Desenhado para o movimento. São Paulo: Nike do Brasil, 2013.

24. Coelho CF, Burini RC. Atividade física para prevenção e tratamento das doenças crônicas não transmissíveis e da incapacidade funcional. Rev Nutr. 2009;22(6):937-46.

25. Uchoga LAR, Altman H. Educação física escolar e relações de gênero: diferentes modos de participar e arriscar-se nos conteúdos de aula. Ver BrasCiên Esporte. 2016;38(2):163-70.

26. Sirard JR, Pfeiffer KA, Pate RR. Motivational factors associed with sports program participation in middle school students. J Adolec Health. 2006;38(6):696-703.

27. Casey MM, Elime RM, Payne WR, Harvey JT. Using a socioecological approach to examine participation in sport and physical activity among rural adolescent girls. Qual Health Res. 2009;19(7):881-93.

Recebido: 20/02/2021

Aprovado: 22/12/2021

\section{Como citar este artigo:}

Silva IJO, Coelho-Ravagnani CF, Tenório MCM, Tassitano RM, Ravagnani FCP. A estratégia LET US Play aumenta a atividade física de crianças nas aulas de educação física escolar. Rev Bras Ativ Fis Saúde. 2021;26:e0238. DOI: 10.12820/rbafs.26e0238 